\title{
Impaired immune function: An early marker for cancer cachexia
}

\author{
JOYCE FABER ${ }^{1,4}$, ARJAN P. VOS ${ }^{1}$, DIANE KEGLER ${ }^{1}$, JOSEP ARGILÉS ${ }^{2}$, \\ ALESSANDRO LAVIANO $^{3}$, JOHAN GARSSEN ${ }^{1,4}$ and ARDY VAN HELVOORT ${ }^{1}$ \\ ${ }^{1}$ Danone Research, Centre for Specialised Nutrition, Wageningen, The Netherlands; ${ }^{2}$ Cancer Research Group, \\ Departament de Bioquímica i Biologia Molecular, Facultat de Biologia, Universitat de Barcelona, Spain; ${ }^{3}$ Department \\ of Clinical Medicine, University La Sapienza, Rome, Italy; ${ }^{4}$ Department of Pharmacology and Pathophysiology, \\ Utrecht Institute for Pharmaceutical Sciences (UIPS), Utrecht University, The Netherlands
}

Received August 4, 2009; Accepted September 7, 2009

DOI: 10.3892/or_00000581

\begin{abstract}
Cachexia and chronic inflammation are major challenges for cancer patients, leading to serious consequences. Accordingly, it is of high clinical relevance to identify early risk factors for optimal treatment, as these are currently not available. The present study demonstrates a strong decline in contact hypersensitivity, a parameter for cell-mediated immunity, in tumor-bearing cachectic mice. Interestingly, a significant reduction was already observed during the pre-cachectic state, reflecting an impaired immune function prior to weight loss. Extrapolating to the human setting, reduced immune competence of cancer patients could serve as an early marker for cancer cachexia, enabling an early supportive care strategy.
\end{abstract}

\section{Introduction}

Cancer cachexia occurs in the majority of cancer patients and is a major contributor to morbidity and mortality in advanced disease $(1,2)$. Although there is still debate on the definition of cachexia, characteristics of this chronic condition of catabolism include, progressive, involuntary weight loss, wasting, anorexia, asthenia and fatigue (1-3). Tumor-derived factors, therapeutic strategies, but also nutritional status, age and even stress and depression are involved in this process, resulting in a chronic inflammatory state and impaired immune responsiveness $(1,4,5)$.

Immune suppression is a major problem in these cancer patients leading to disease progression, increased complications and a delayed or suboptimal treatment protocol (e.g., surgery, chemotherapy, radiotherapy) resulting in a reduced

Correspondence to: Dr Joyce Faber, Danone Research, Centre for Specialised Nutrition, P.O. Box 7005, 6700 CA Wageningen, The Netherlands

E-mail: joyce.faber@danone.com

Key words: cancer, immune function, cachexia, inflammation, nutrition quality of life and a poor prognosis (4-7). The dysfunction of the immune system involves multiple mechanisms and is in humans characterized by a reduction of monocyte-, macrophage- and dendritic cell (DC)-function and NK cell activity, leading to an increased risk of infections and a poor clinical outcome $(4,5,8,9)$.

Prevention and treatment of cancer cachexia should be recognized as an integral part of cancer therapy. It might stop or reverse the nutritional decline and counteract dysfunction of the immune system in order to improve clinical outcome and quality of life $(10,11)$. However, recent findings show that significant metabolic, biochemical and molecular changes on muscle proteolysis already occur in patients before any evidence of body weight loss (12). Consequently, it is of high clinical relevance to investigate the appearance of immune suppression in the course of the cachexia process as well, in order to identify early risk factors.

A recently modified C26 tumor model was used to study the cachectic process and immune competence. Cachexia was induced by inoculation of murine colon adenocacinoma (C26) cells in syngenic CD2F1 mice leading to several cachectic features (13-17). To measure immune competence contact hypersensitivity (CHS) towards oxazolone was measured as a validated in vivo parameter for Th1-mediated immune function. Th1 immunity is involved in anti-tumor immune responses, but plays a pivotal role in the defence against infections with pathogenic bacteria and viruses as well. CHS was measured at two time-points during the study, to observe changes in the immune status in a pre-cachectic and a cachectic state of the mice.

\section{Materials and methods}

Animals and diets. Six to seven-week old syngenic male CD2F1 mice (BALB/c x DBA/2) were obtained from Harlan Nederland (Horst, The Netherlands). All experimental procedures were approved by the Animal Experimental Committee and complied with the principles of laboratory animal care. Animals were housed individually in a climatecontrolled animal care facility with a constant room temperature and humidity. All animals had free access to food and drinking water. Upon arrival animals were acclimatized for one week and subsequently randomized on basis of body- 


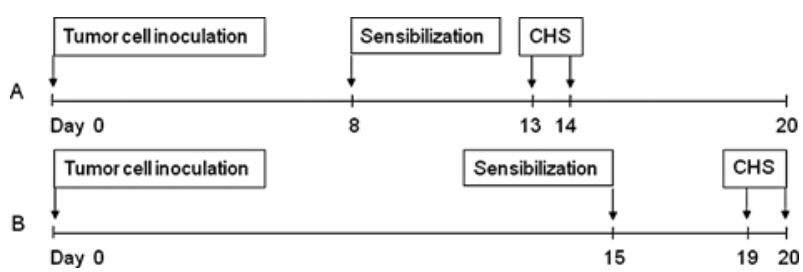

Figure 1. Experimental set-up. CHS against oxazolone is measured in a precachectic state at day 13/14 (A) and in a cachectic state at day 19/20 (B).

weight. The experiments were divided in: experiment A, designed to investigate the effect on immune function in a pre-cachectic state and experiment $\mathrm{B}$, designed to investigate the effect on immune function in a cachectic state of the mice. In both experiments $\mathrm{A}$ and $\mathrm{B}$, mice were divided into a control group (C) and a tumor-bearing group (TB). Both groups received a control diet based on AIN93-M (Research Diet Services, Wijk bij Duurstede, The Netherlands), supplied as pellets and contained per $\mathrm{kg}$ food: $126 \mathrm{~g}$ protein $(100 \%$ casein), $727 \mathrm{~g}$ carbohydrates and $40 \mathrm{~g}$ fat (100\% soy oil).

Experimental design. Murine colon-26 adenocarcinoma cells were used to induce cachexia in mice. Shortly, on day 0 tumor cells $\left(5 \times 10^{5}\right.$ cells in $\left.0.2 \mathrm{ml}\right)$ were inoculated, under general anaesthesia (isoflurane $/ \mathrm{N}_{2} \mathrm{O} / \mathrm{O}_{2}$ ), subcutaneously into the right inguinal flank of $\mathrm{CD} 2 \mathrm{~F} 1$ mice in the tumor-bearing group. Animals in the control group received a sham injection with $0.2 \mathrm{ml} \mathrm{HBSS}$. Body weight (BW), food intake and tumor size (length and width) were measured three times a week. To investigate effects on the immune system contact hypersensitivity (CHS) against oxazolone was determined, as an in vivo model for cellular (Th1-dependent) immunity (Fig. 1). In experiment $\mathrm{A}, \mathrm{CHS}$ was measured in a pre-cachectic state of the mice, while in experiment B CHS was determined when the mice were already cachectic (Fig. 1). Briefly, on day 8 (experiment A) or day 15 (experiment B) all animals were sensitized with $150 \mu 13 \%$ oxazolone solution [4-ethoxymethylene-2-phenyl-2-oxazolin-5-one (Sigma-Aldrich Chemie, Zwijndrecht, The Netherlands), $300 \mathrm{mg}$ in $7.5 \mathrm{ml} 96 \%$ ethanol and $2.5 \mathrm{ml}$ acetone] applied on their shaved breast and abdomen. Subsequently, at day 13 (experiment A) or day 19 (experiment B) ear thickness was measured under general anaesthesia and all animals were hapten challenged with $25 \mu 1$ $0.8 \%$ oxazolone solution ( $32 \mathrm{mg}$ in $3 \mathrm{ml} 96 \%$ ethanol and $1 \mathrm{ml}$ acetone) topical to the ear pinnae. At day 14 (experiment A) or day 20 (experiment B) after tumor inoculation $(24 \mathrm{~h}$ after the challenge), ear swelling was measured under general anaesthesia to determine the Th1 immune response. In both experiments A and B mice were sacrificed at day 20 and tumor, spleen, thymus, fat and skeletal muscles [m. Extensor Digitorum Longus (mEDL) and m. Tibialis Anterior (mTA)] were dissected and weighed.

Statistics. All data were expressed as means \pm SEM. Statistical analysis was performed using SPSS 12.0.1 (SPSS Benelux, Gorinchem, The Netherlands). The effect of tumor inoculation was tested using a Student's t-test when data were normally distributed. A non-parametric Mann Whitney U test was per-

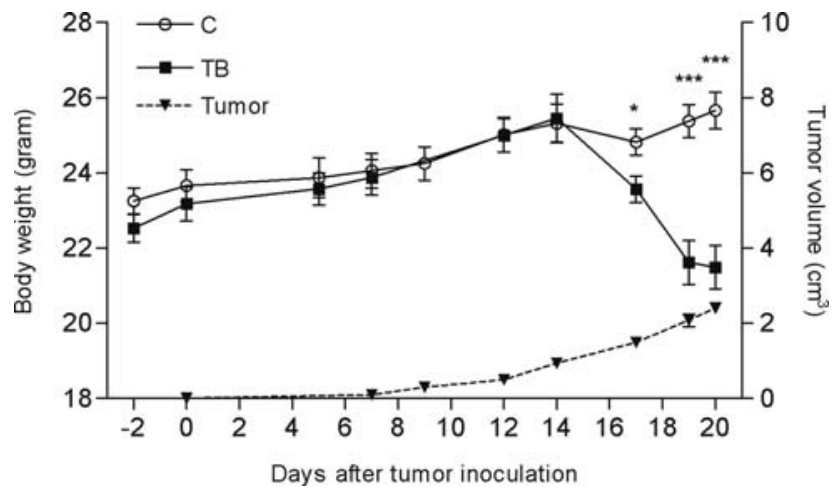

Figure 2. Effects of tumor inoculation on body weight. Left y-axes, data represent means \pm SEM of bodyweight (gram) in control (C) group $(n=10)$ and tumor-bearing (TB) group $(n=9)$ during the experiment. *Significantly different $(\mathrm{p}<0.05)$ from control group $(\mathrm{C}){ }^{*}{ }^{* * *}$ Significantly different $(\mathrm{p}<0.001)$ from control group (C). Right $y$-axes, data represent means \pm SEM of tumor volume $\left(\mathrm{cm}^{3}\right)$ in the tumor-bearing $(\mathrm{TB})$ group $(\mathrm{n}=9)$ during the experiment.

Table I. Physiological cachexia and immune parameters at day 20 .

$\mathrm{C}$ TB

\begin{tabular}{lcc}
\hline Cachexia & & \\
Body weight $(\mathrm{g})$ & $25.7 \pm 0.5$ & $21.5 \pm 0.6^{\mathrm{a}}$ \\
Tumor weight $(\mathrm{g})$ & $0.0 \pm 0.0$ & $2.6 \pm 0.2^{\mathrm{a}}$ \\
Carcass weight $(\mathrm{g})$ & $25.7 \pm 0.5$ & $18.9 \pm 0.5^{\mathrm{a}}$ \\
Fat $(\mathrm{g})$ & $4.6 \pm 0.3$ & $2.5 \pm 0.1^{\mathrm{a}}$ \\
m. Extensor Digitorum Longus $(\mathrm{mg})$ & $12.1 \pm 0.5$ & $7.8 \pm 0.4^{\mathrm{a}}$ \\
Longus (mg) & & \\
m. Tibialis Anterior $(\mathrm{mg})$ & $46.8 \pm 1.2$ & $32.2 \pm 0.9^{\mathrm{a}}$
\end{tabular}

Immune

$\begin{array}{lll}\text { Thymus weight }(\mathrm{mg}) & 34.3 \pm 2.4 & 18.4 \pm 1.6^{\mathrm{a}}\end{array}$

Spleen weight $(\mathrm{mg}) \quad 115.5 \pm 4.8225 .1 \pm 18.4^{\mathrm{a}}$

Data represent means \pm SEM of control $(C)$ group $(n=10)$ and tumorbearing $(T B)$ group $(n=9)$. aSignificantly different $(\mathrm{p}<0.001)$ from control group (C).

formed when data were not normally distributed. Differences were considered significant at $\mathrm{p}<0.05$.

\section{Results}

Animals in both the control (C) and tumor-bearing (TB) group demonstrated a normal growth in bodyweight (BW) until day 14 (Fig. 2). Afterwards, a very strong reduction of $\mathrm{BW}$ was observed in the TB compared to the $\mathrm{C}$ group, which was statistically significant from day $17(\mathrm{p}<0.05)$ to day 20 $(\mathrm{p}<0.001$, Fig. 2). The tumor induced progressive cachectic state of the mice was confirmed by a significant reduction in carcass weight, fat weight and skeletal muscle weight at day $20(\mathrm{p}<0.001$, Table I), while food intake was not affected. At 


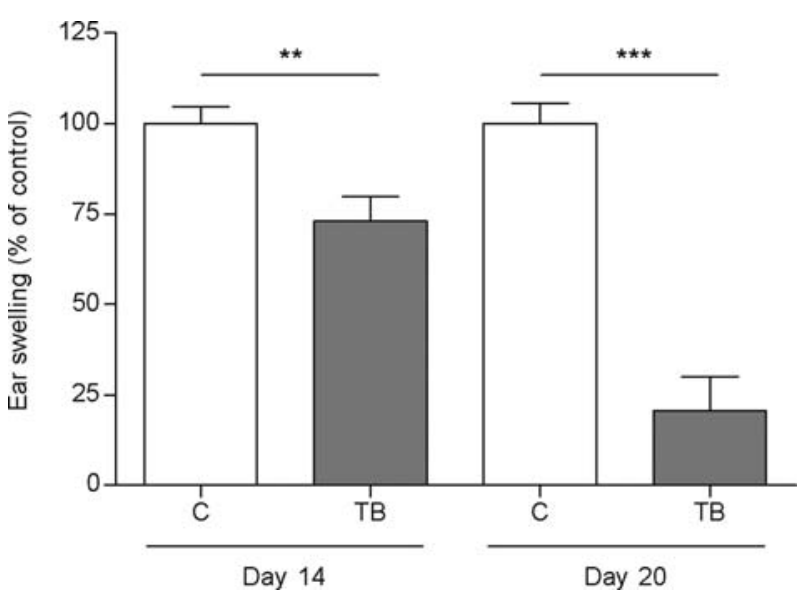

Figure 3. Effects of tumor inoculation on contact hypersensitivity in precachectic and cachectic mice. Data represent means \pm SEM of ear swelling in control (C) group $(n=10)$ and tumor-bearing $(T B)$ group $(n=9)$ at day 14 (pre-cachectic state) and day 20 (cachectic state). ${ }^{* *}$ Significantly different $(\mathrm{p}<0.01)$ from control group $(\mathrm{C}) .{ }^{* * *}$ Significantly different $(\mathrm{p}<0.001)$ from control group (C).

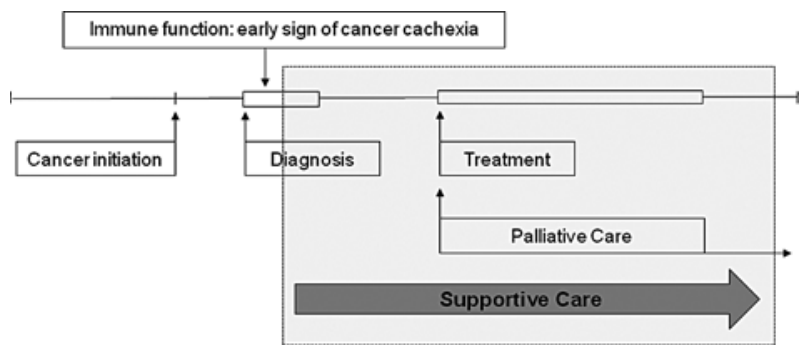

Figure 4. Supportive care strategy. Proposed multi-disciplinary supportive care strategy, which is initiated at the moment of diagnosis and runs parallel to the pathway of cancer therapies.

this time-point cachectic mice demonstrated a very strong decline in immune competence as measured by CHS $(79.4 \%$, $\mathrm{p}<0.001$, Fig. 3). However, CHS responses were not affected in cachectic mice only, already during the pre-cachectic state a significant reduced ear swelling has been observed (27.0\%, $\mathrm{p}<0.01$, Fig. 3), reflecting an impaired immune function in tumor-bearing mice already prior to weight loss.

\section{Discussion}

The reduced immune function at day 20 could be explained by the cachectic and chronic inflammatory state of the mice. The pro-inflammatory cytokines interleukin (IL)-1ß, IL-6, tumor necrosis factor (TNF)- $\alpha$ and interferon (IFN)- $\gamma$ and the eicosanoid $\mathrm{PGE}_{2}$ play a major role in the impaired immune response to the tumor $(1,8)$, but also to pathogens leading to an increased number and severity of infections. At day 14, pro-inflammatory cytokines may already play a role in this process, but the absence of cachectic features suggests that other mechanisms are involved as well. Animal studies, but also human clinical trials describe the presence of myeloid cells involved in the initiation of the inflammatory process. Circulating monocytes are recruited to the tumor site by inflammation associated chemokines and cytokines and differentiate into tumor-associated macrophages (TAM) $(8,18)$. TAM exacerbate the inflammatory response at the tumor environment, further driving forward the malignancy cascade (8). Recent published data describe the relationship between inflammation and metastasis as well (19). Simultaneously, a population of suppressor cells called myeloid derived suppressor cells (MDSC) is induced by pro-inflammatory cytokines $(18,20)$. MDSC are found in many patients and experimental animals with cancer and cause a profound immune suppression (20).

The currently presented effects on immune function prior to weight loss may have important clinical implications when extrapolated to the human setting. Because the lack of biomarkers of a pre-cachectic state, immune function parameters, such as CHS, could serve as an early predictive marker for cancer cachexia which can be measured in cancer patients at risk for cachexia. The possibility to implement such an early marker would consequently enable an early supportive care strategy in these patients including specific nutritional interventions. The addition of immune modulatory ingredients might be an interesting opportunity for intervention, leading to a reduced inflammatory state, improved immune responsiveness and consequently, an improved performance status (10). Early provision of nutritional support might improve an effective management of cachexia related health factors as well. Every cancer patient should be regarded as a potential candidate to develop immune dysfunction and other cachexia related features. Therefore, a multi-disciplinary approach (12) is recommended, which is initiated at the moment of diagnosis and runs parallel to the pathway of cancer therapies (Fig. 4).

In conclusion, the immune competence of cancer patients should be monitored in early stages of the disease, since impaired immune function could serve as an early marker for cancer cachexia. Consequently, supportive care should be provided at the earliest time-point possible, to maximize the chance of reducing disease progression, reducing the frequency and severity of complications and improving treatment adherence.

\section{Acknowledgements}

The authors would like to thank Dr Rob Verdooren for his advice on the statistical analysis, Dr Marchel Gorselink for his experimental advice and Donna McCarthy for the kind gift of the C26 cell line. The authors declare that there is no role of the funding source.

\section{References}

1. Ross JA and Fearon KC: Eicosanoid-dependent cancer cachexia and wasting. Curr Opin Clin Nutr Metab Care 5: 241-248, 2002.

2. Van Cutsem E and Arends J: The causes and consequences of cancer-associated malnutrition. Eur J Oncol Nurs 9 (Suppl. 2): S51-S63, 2005.

3. Argiles JM, Alvarez B and Lopez-Soriano FJ: The metabolic basis of cancer cachexia. Med Res Rev 17: 477-498, 1997.

4. Evans C, Dalgleish AG and Kumar D: Review article: immune suppression and colorectal cancer. Aliment Pharmacol Ther 24: 1163-1177, 2006.

5. Hadden JW: Immunodeficiency and cancer: prospects for correction. Int Immunopharmacol 3: 1061-1071, 2003. 
6. Young MR: Eicosanoids and the immunology of cancer. Cancer Metastasis Rev 13: 337-348, 1994.

7. Herber DL, Nagaraj S, Djeu JY and Gabrilovich DI: Mechanism and therapeutic reversal of immune suppression in cancer. Cancer Res 67: 5067-5069, 2007.

8. Ben-Baruch A: Inflammation-associated immune suppression in cancer: the roles played by cytokines, chemokines and additional mediators. Semin Cancer Biol 16: 38-52, 2006.

9. Whiteside TL: Immune suppression in cancer: effects on immune cells, mechanisms and future therapeutic intervention. Semin Cancer Biol 16: 3-15, 2006.

10. Van Bokhorst-De van der Schueren MA: Nutritional support strategies for malnourished cancer patients. Eur J Oncol Nurs 9 (Suppl. 2): S74-S83, 2005.

11. Nitenberg G and Raynard B: Nutritional support of the cancer patient: issues and dilemmas. Crit Rev Oncol Hematol 34: $137-168,2000$

12. Muscaritoli M, Costelli P, Aversa Z, Bonetto A, Baccino FM and Rossi Fanelli F: New strategies to overcome cancer cachexia: from molecular mechanisms to the 'Parallel Pathway'. Asia Pac J Clin Nutr 17 (Suppl. 1): S387-S390, 2008.

13. Tanaka Y, Eda H, Tanaka T, et al: Experimental cancer cachexia induced by transplantable colon 26 adenocarcinoma in mice. Cancer Res 50: 2290-2295, 1990.

14. Strassmann G, Fong M, Kenney JS and Jacob CO: Evidence for the involvement of interleukin 6 in experimental cancer cachexia. J Clin Invest 89: 1681-1684, 1992.
15. Strassmann G, Jacob CO, Evans R, Beall D and Fong M: Mechanisms of experimental cancer cachexia. Interaction between mononuclear phagocytes and colon-26 carcinoma and its relevance to IL-6-mediated cancer cachexia. J Immunol 148: 3674-3678, 1992.

16. Tanaka M, Miyazaki H, Takeda Y and Takeo S: Detection of serum cytokine levels in experimental cancer cachexia of colon 26 adenocarcinoma-bearing mice. Cancer Lett 72: 65-70, 1993.

17. Soda K, Kawakami M, Kashii A and Miyata M: Characterization of mice bearing subclones of colon 26 adenocarcinoma disqualifies interleukin- 6 as the sole inducer of cachexia. Jpn J Cancer Res 85: 1124-1130, 1994.

18. Sica A and Bronte V: Altered macrophage differentiation and immune dysfunction in tumor development. J Clin Invest 117: 1155-1166, 2007.

19. Mantovani A: Cancer: Inflaming metastasis. Nature 457: 36-37, 2009.

20. Bunt SK, Yang L, Sinha P, Clements VK, Leips J and OstrandRosenberg S: Reduced inflammation in the tumor microenvironment delays the accumulation of myeloid-derived suppressor cells and limits tumor progression. Cancer Res 67: 10019-10026, 2007. 